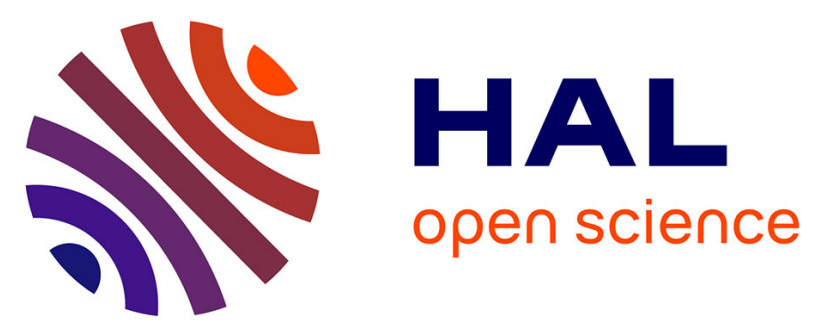

\title{
Quantitative assessment and prediction of the contact area development during spherical tip indentation of glassy polymers.
}

Christophe Pelletier, Jaap M.J. den Toonder, Leon E. Govaert, Norio Hakiri, Mototsugu Sakai

\section{To cite this version:}

Christophe Pelletier, Jaap M.J. den Toonder, Leon E. Govaert, Norio Hakiri, Mototsugu Sakai. Quantitative assessment and prediction of the contact area development during spherical tip indentation of glassy polymers.. Philosophical Magazine, 2008, 88 (09), pp.1291-1306. 10.1080/14786430802123224 . hal-00513894

\section{HAL Id: hal-00513894 \\ https://hal.science/hal-00513894}

Submitted on 1 Sep 2010

HAL is a multi-disciplinary open access archive for the deposit and dissemination of scientific research documents, whether they are published or not. The documents may come from teaching and research institutions in France or abroad, or from public or private research centers.
L'archive ouverte pluridisciplinaire HAL, est destinée au dépôt et à la diffusion de documents scientifiques de niveau recherche, publiés ou non, émanant des établissements d'enseignement et de recherche français ou étrangers, des laboratoires publics ou privés. 


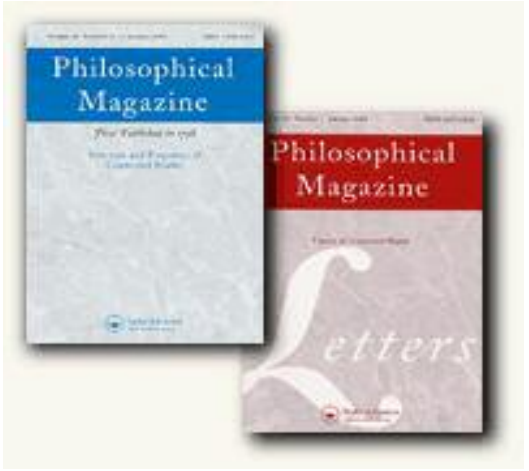

\section{Quantitative assessment and prediction of the contact area development during spherical tip indentation of glassy polymers.}

\begin{tabular}{|r|l|}
\hline Journal: & Philosophical Magazine \& Philosophical Magazine Letters \\
\hline Manuscript ID: & TPHM-07-Aug-0230.R1 \\
\hline Journal Selection: & Philosophical Magazine \\
\hline Author: & 09-Apr-2008 \\
\hline Complete List of Authors: & $\begin{array}{l}\text { Pelletier, Christophe; Eindhoven University of Technology, Materials } \\
\text { Research Institute } \\
\text { den Toonder, Jaap; Eindoven University of Technology, Materials } \\
\text { Technology Institute; Philips Research } \\
\text { Govaert, Leon; Eindhoven University of Technology, Materials } \\
\text { Research Institute } \\
\text { Hakiri, Norio; Toyohashi University of Technology, Materials Science } \\
\text { Sakai, Mototsugu; Toyohashi University of Technology, Materials } \\
\text { Science }\end{array}$ \\
\hline Keywords: & indentation, polymers \\
\hline Keywords (user supplied): & contact-area, pile-up, mechanical-properties \\
\hline &
\end{tabular}

\section{(5) ScholarONE" \\ Manuscript Central}




\title{
Quantitative assessment and prediction of the contact area development during spherical tip indentation of glassy polymers.
}

\author{
C.G.N PELLETIER $\dagger$, J.M.J. DEN TOONDER $* \dagger$, L.E. GOVAERT $\dagger$, N. HAKIRI $\$$, M. SAKAI $\ddagger$ \\ $\dagger$ Materials Technology Institute (MaTe), Eindhoven University of Technology, P.O. Box 513, NL-5600MB, \\ Eindhoven, The Netherlands \\ \$ Department of Material Science, Toyohashi University of Technology, Tempaku-cho, Toyohashi 441-8580, \\ Japan \\ *Corresponding author. Email: j.m.j.d.toonder@tue.nl \\ Abstract \\ In this paper we study the development of the contact area during indentation of polycarbonate. The contact area was measured in \\ situ using an instrumented indentation microscope and compared with numerical simulations using an elasto-plastic constitutive \\ model. The parameters in the model were obtained using macroscopic tests. Indentations were performed on samples with different \\ thermal histories and at different speeds. For all cases, the numerical model correctly predicted the development of the contact area \\ during indentation. For increasing strain rates, the contact area decreases at equal indentation depths. Annealing the samples results \\ in a smaller contact area at equal indentation depth. Using only numerical simulation, it was also shown that pile-up around the \\ indenter results from localization effects and is thus promoted by strain softening properties of the indented material. Strain \\ hardening, on the other hand, will tend to promote sink-in. Finally, we performed simulations of load-relaxation during indentation. \\ The results indicate that about $40 \%$ of the total observed relaxation may be assigned to plastic effects.
}

Keywords: indentation; contact area; pile-up; glassy polymers; mechanical properties 


\section{Introduction}

Nowadays, indentation is a fairly mature technique to probe mechanical properties of small volumes of materials and especially thin films. The main requirement for an indentation sample is that the indented surface should be sufficiently smooth to obtain reproducible results, but no further special sample preparation is needed. During indentation, the applied load and the tip displacement are continuously recorded. From such data mechanical properties, like the elastic modulus or the yield stress of the materials, can be extracted using various methods depending on the deformation regime and tip's geometry. In all indentation analysis models, the accuracy of the estimated mechanical properties is strongly related to the accurate determination of the contact area between the indenter tip and the material surface, which is not always straightforward. The main difficulty is that for most materials the contact area cannot be estimated directly from the measured tip indenter displacement. Figure 1 illustrates the fact that the measured displacement of the tip $(h)$ is in practice hardly ever equal to the contact depth $\left(h_{c}\right) . h_{c}$ is the depth over which the tip and the surface make contact, and knowing the tip's geometrical shape this determines the (projected) contact area. At the vicinity of the tip, the surface can sink-in (on the right in figure 1) or pile-up (on the left in figure 1).

[Insert figure 1 about here]

For the special case of frictionless contact of a spherical indenter with a flat linearly elastic half space, the surface will sink-in in such a way that the contact depth $\left(h_{c}\right)$ is equal to half of the tip displacement $h$. For small displacements with respect to the tip size (radius), the projected contact area for spherical indentation is given by [1]:

$$
A_{p}=\pi a^{2} \approx \pi R h
$$

where $A_{p}$ is the projected area, a is the contact radius, $R$ is the tip radius and $h$ is the tip displacement defined by:

$$
h=h_{p}+h_{c}
$$

where $h_{c}$ is the contact depth and $h_{p}$ the difference between the displacement and the contact depth (see figure $1)$.

In the general case, the projected contact area is defined as a function of the contact depth by a simple geometric relation:

$$
A_{p}=\pi a^{2}=\pi\left(2 R-h_{c}\right) h_{c}
$$

where $h_{c}$ is the contact depth. Unfortunately, when in addition to elastic deformation, also plastic deformation occurs, no analytical solution exists that describes the relationship between the actual contact depth and the experimentally measured tip displacement. Therefore the contact area cannot be determined directly from the load-displacement curves, which makes it impossible to determine the mechanical properties without assumptions or approximations about the contact area.

Popular methods to determine mechanical properties from indentation were proposed by Oliver and | Pharr for a Berkovich tip \2〕 and by Field and Swain for a spherical tip [3]. Essentially, the method is based on elastic contact theory of symmetric indenters. Plastic deformation, occurring in addition to purely elastic strains, is assumed to result only in a shift of the total indentation depth and not in a change of the nature of the surface deformation near the contact. The final analysis, therefore, is a purely elastic analysis of the 
(elastic) unloading part of the indentation curve. In principle, the method is thus not applicable to materials exhibiting pile-up or extensive sink-in. In daily practice, calibration procedures involving the fitting of a polynomial function, that describes the relationship between the projected contact area and the indentation depth, indirectly compensate for this flaw in the method. However this compensation is only valid for the material used for the calibration, which is usually fused silica.

Pile-up, which may occur in many metals and in polymers, can lead to significant errors in the determination of the contact area. Using finite element simulations, it was shown that the Oliver and Pharr method may significantly underestimate the contact area when the material piles-up [4]. The amount of pileup depends on the ratio of the elastic modulus to the yield stress and on work hardening properties. For a large ratio and no or little work hardening, the material will exhibit a pronounced pile-up. In contrast, an increase in work hardening will tend to inhibit the pile-up. Compared to Berkovich indentation, the situation is more complex for spherical indentation as the amount of pile-up is a function of the indentation depth $[5]$, Some methodologies, alternative to that of Oliver and Pharr, attempt to account for the lack of knowledge of the true contact area in instrumented indentation [6-8], in particular for metals. In these approaches, however, the problem is that a single indentation load-displacement curve can have multiple solutions for the mechanical properties, except for special cases. A unique solution of the problem, again, can only be obtained if the actual evaluation of the contact area is known.

It should be mentioned, that the numerical model used in reference [5] to study the pile-up is based on an elasto-plastic constitutive model with strain hardening, which is appropriate for a class of metals. Compared to metals, glassy polymers possess a more complex intrinsic behaviour, namely a more pronounced strain rate dependence of the yield stress and a post yield region displaying strain softening as well as strain hardening. These intrinsic properties result in an evolution of the contact area during indentation that cannot be described with common metal models since these do not capture these specific intrinsic features.

In the last 20 years, considerable effort has been made to model the post yield behaviour of glassy polymers and a number of 3D numerical models were developed and validated, by Boyce et al. at MIT [9-11] Paul Buckley et al. in Oxford [12-14] and our own group in Eindhoven [15-17]. Common factors of these models are the application of a stress dependent viscosity to capture the deformation kinetics and the use of rubber elasticity to model strain hardening.

We will use our numerical model to investigate the evolution of the contact area during spherical indentation on polycarbonate. The simulations will be validated using a recently developed technique, the | instrumented indentation microscope $[18,19 \downarrow$ which enables the direct measurement of the contact area during indentation.

\section{Mechanical properties of glassy polymers: phenomenology}

Intrinsic behaviour is defined as the response of the material under homogenous deformation. In general the intrinsic behaviour of amorphous polymers, like polycarbonate, polystyrene, or polymethylmethacrylate, exhibits a non-linear viscoelastic regime at low deformation followed by yielding and complex post yield behaviour. After yielding, the true stress drops with increasing the plastic strain (strain softening) and finally increase again at larger deformation (strain hardening).

This response is dependent on temperature, pressure and thermodynamic state. An illustration of the influence of deformation rate on polycarbonate $(\mathrm{PC})$ is shown in figure $2 \mathrm{a}$. With an increasing strain rate the yield stress increases leading to a shift of the total plastic response to higher stress. Contrary to the yield stress, the strain softening is rate independent. Figure $2 \mathrm{~b}$ shows the influence of the material's thermal history on the plastic response. By annealing the sample, the yield stress and the strain softening will increase. It should be noticed that the response at large deformations is independent of the thermal history. At large deformation, namely, the effect (of the thermal-history) has been erased by the plastic deformation, i.e. the 
material returns to the rejuvenated state. In such a state, the intrinsic response of amorphous polymers exhibits no strain softening as is illustrated in the figure.

[Insert figure 2 about here]

\section{Modelling}

\subsection{Constitutive modelling}

In previous work we developed an 3D elasto-viscoplastic constitutive equation that accurately captures the deformation characteristics of polymer glasses $[15,16,20,21]$. The basis of this constitutive model is the division of the total stress into two contributions, first proposed by Howard and Thackray [22];

$$
\sigma=\sigma_{\mathrm{s}}+\sigma_{\mathbf{r}}
$$

Deleted: $[12,13,17,18]$

Deleted: a

Deleted: [19]

Deleted: $[13,20]$

where $G_{r}$ is the strain hardening modulus, $\widetilde{\mathbf{B}}$ is the isochoric left-Cauchy-Green deformation tensor, and the superscript d denotes that we take the deviatoric part.

The so-called driving stress $\boldsymbol{\sigma}_{\mathrm{s}}$ in equation (4) accounts for the rate-dependent plastic flow response, attributed to intermolecular interactions on a segmental scale $[15,21]$ and is represented by a compressible Leonov

model [20, 24];

$$
\boldsymbol{\sigma}_{\mathbf{s}}=K(\mathbf{J}-1) \mathbf{I}+G \widetilde{\mathbf{B}}_{\mathbf{e}}^{\mathbf{d}}
$$

where $K$ is the bulk modulus and $G$ is the shear modulus. The relative volume change $\mathbf{J}$ and the isochoric elastic left Cauchy Green deformation tensor $\widetilde{\mathbf{B}}_{e}$ are implicitly given by:

$$
\begin{gathered}
\dot{\mathbf{J}}=\mathbf{J} \operatorname{tr}(\mathbf{D}) \\
\widetilde{\mathbf{B}}_{e}=\left(\mathbf{D}^{\mathbf{d}}-\mathbf{D}_{\mathbf{p}}^{\mathbf{d}}\right) \widetilde{\mathbf{B}}_{\mathbf{e}}+\widetilde{\mathbf{B}}_{\mathbf{e}}\left(\mathbf{D}^{\mathbf{d}}-\mathbf{D}_{\mathbf{p}}\right)
\end{gathered}
$$

The left hand side of equation (8) represents the (objective) Jaumann derivative of the isochoric elastic left

| Cauchy Green tensor. The tensor $\mathbf{D}$ denotes the rate of deformation tensor, and $\mathbf{D}_{\mathbf{p}}$ is the plastic rate of deformation tensor. To complete the constitutive description the plastic deformation rate is expressed in the Cauchy stress tensor by a generalized non-Newtonian flow rule:

$$
\mathbf{D}_{\mathbf{p}}=\frac{\boldsymbol{\sigma}_{\mathbf{s}}^{\mathbf{d}}}{2 \eta\left(\bar{\tau}, p, S_{a}\right)}
$$

Deleted: $[12,18]$

Deleted: $[17,21]$
Deleted: the 
Under isothermal conditions the nonlinearity of the model is completely governed by the stress-, pressure- and

| state-dependent viscosity $\eta$, defined as [21].

$$
\eta(\bar{\tau}, p, S)=\eta_{o, r} \underbrace{\frac{\bar{\tau} / \tau_{0}}{\sinh \left(\bar{\tau} / \tau_{0}\right)}}_{\text {(I) }} \underbrace{\exp \left(\frac{\mu p}{\tau_{0}}\right)}_{\text {(II) }} \underbrace{\exp \left(S_{a}(t) R_{\gamma}\left(\bar{\gamma}_{p}\right)\right)}_{\text {(III) }}
$$

Here the parameter $\eta_{o, r}$ represents the zero-viscosity for the completely rejuvenated (unaged) state. The part marked (I), with $\bar{\tau}$ being the equivalent stress $\_25$, represents the stress dependent part of the viscosity governed by the parameter $\tau_{o}$. Part (II) is the pressure dependent part, governed by the pressure dependence parameter $\mu$ and the hydrostatic pressure $p[26]$. The combination of part (I) and (II) gives a rate dependent plastic flow response according to the pressure modified Eyring flow expression $\lceil 27,28$, Finally, part (III) represents the dependence of the viscosity on physical aging and rejuvenation (strain softening) this on the temperature- and deformation history. The parameter $S_{a}$ can be regarded as a state parameter that uniquely determines the current state of the material. Evolution of $S_{a}$ with time allows us to capture the change of mechanical properties over time as a result of physical aging. In the present investigation, we will only consider materials with different initial $S_{a}$ values (obtained by application of different thermal histories).

The function $R_{\gamma}$ in equation (10) describes the strain softening process that is the erasure of thermal history by plastic deformation. It is expressed as:

$$
R_{\gamma}\left(\bar{\gamma}_{p}\right)=\frac{\left(1+\left(r_{o} \cdot \exp \left(\bar{\gamma}_{p}\right)\right)^{r_{1}}\right)^{\frac{r_{2}-1}{r_{1}}}}{\left(1+r_{o}^{r_{1}}\right)^{\frac{r_{2}-1}{r_{1}}}}
$$

where $r_{0}, r_{l}$ and $r_{2}$ are fitting parameters, and $\bar{\gamma}_{p}$ denotes the equivalent plastic strain [29].

The essence of the influence of physical aging and of strain softening, modelled by the state parameter $S_{a}$ (equation (10)), is illustrated in figure 3 a which shows the strain-rate dependence of the yield stress resulting from equation (10). In the reference state, i.e. the fully rejuvenated state, the parameter $S_{a}$ will be initially equal to zero. With physical aging (also during processing) the value of $S_{a}$ will increase, which leads to a shift of the yield stress- strain rate along the $\log$ (strain rate) axis. At a constant strain rate, the result will be an increase in yield stress compared to that of the rejuvenated state. Upon deformation, the increasing equivalent plastic strain $\bar{\gamma}_{p}$ triggers strain softening (equation (11)) and the yield stress shifts back to that of the rejuvenated state. As a result of rejuvenation due to deformation, the yield stress drops with increasing strain and the intrinsic stress-strain curve evolves back to that of the rejuvenated state at large strains (see figure $3 b)$.

\section{[Insert figure 3 about here]}

For polycarbonate (PC) the parameters in the model (see table 1「211) proved to be independent of the molecular weight distribution and the key parameter, needed to adjust for differences in thermal history (illustrated in figure $2 \mathrm{~b}$ ), is the initial value of the state parameter $S_{a}$.

[Insert table 1 about here]
Deleted: [22]

Deleted: [23]

Deleted: $[24,25]$

Deleted: [26]

Deleted: [18]

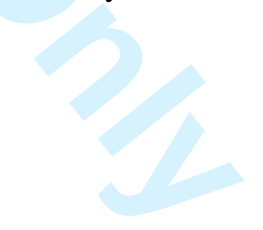




\subsection{Indentation modelling}

In the present model, the viscoelastic elastic regime is approximated with compressible, linear-elastic behaviour. To correctly describe post-yield response, the elastic modulus is generally chosen such that the yield strain is accurately predicted, see figure $4 \mathrm{a}$. This is justified for problems in which large strains are | predominant. For polycarbonate, this yields a value of $900 \mathrm{MPa}$ [17] which is much smaller than the 2200 $2400 \mathrm{MPa}$ generally observed. For indentation, where the elastic deformation will also significantly contribute at larger depth, a drastic underestimation of the material resistance will be obtained when the modulus is chosen too low. Two possibilities are available to solve this problem. The first one is to use a so-called multimode approach to describe the complete pre-yield viscoelastic behaviour. In previous work we showed

| that the behaviour can be accurately captured by a parallel arrangement of eighteen modes [15], Unfortunately, this solution increases tremendously the computation time and will therefore not be used. The second possibility consists of a simple increase of the elastic modulus, which requires adaptation of another single parameter, $\eta_{\mathrm{o}}$, to assure that the post yield response remains identical, see figure $4 \mathrm{~b}$. In that case the modelled yield strain will be smaller than the experimental one. The new set of parameters used for the indentation simulation is presented in table 2.

[Insert figure 4 about here]

[Insert table 2 about here]

Simulations are performed using the Marc / Mentat finite element package. The finite element mesh used for the simulation is depicted in figure 5. Axisymmetric quadrilateral linear elements were used. The total domain shown in figure 5 has dimensions $1 \mathrm{~mm} \times 1 \mathrm{~mm}$ and consists of 2192 nodes and 2061 elements. The mesh becomes more refined towards the region of contact (figure 5) and the size of the elements which come into contact with the indenter is about $300 \mathrm{~nm}$. The indenter was modelled as a rigid sphere with a radius of $R 100 \mu \mathrm{m}$. The contact radius was estimated by the y coordinate of the last node in contact. The accuracy of the contact radius estimate, limited by the discretization, was $0.0015 \bar{R}$, comparable to the experimental accuracy. Since preliminary computations showed, that friction did not influence the results significantly up to indentation depths of $h / R=0.15$, we performed all simulations assuming frictionless contact.

[Insert figure 5 about here]

\section{Materials and experimental methods}

\subsection{Materials}

The material used in this study was polycarbonate (Makrolon, Bayer), obtained in the form of extruded sheet of $3 \mathrm{~mm}$ thickness. Prior to the indentation tests, the sheet roughness was determined by using a commercial Atomic Force Microscope (Digital Instruments, Nanoscope IIIa) operating in tapping mode. A mean roughness, $\mathrm{R}_{\mathrm{a}}$, of about $5 \mathrm{~nm}$ was measured.

From the extruded sheet, samples with a size of $10 \mathrm{~mm}$ x $10 \mathrm{~mm}$ were cut. For half of the samples no further heat treatment was applied prior to indentation. The other half was annealed at $130^{\circ} \mathrm{C}$ for 24 hours in an air circulated oven and subsequently cooled down slowly to room temperature. 


\subsection{Optical indentation microscope}

In order to make in situ observations of the contact area during indentation tests, a unique indentation device was designed where a transparent indenter is coupled with a microscope and a CCD camera (see figure 6) $\square 18$, 191. The tip of the indenter is optically aligned to a microscope and a CCD-video-camera and the contact area is observed through the indenter tip. At the maximum magnification, the inaccuracy in the length measured on video-images is $\pm 0.5 \mu \mathrm{m}$. The depth of penetration $(h)$ is measured by a couple of linear transducers and the indentation load $(P)$ is monitored by a load cell. Here, a diamond cone indenter (the apex angle of $90^{\circ}$ ) with a tip radius $R$ of $100 \mu \mathrm{m}$ was used. Hence the accuracy of the contact radius measurement was $0.005 R$. The used tip geometry offers the possibility to fully exploit the advantage of the axisymmetry condition for the numerical simulation and reduce the number of elements. Using the instrumented indentation microscope, the contact area can be determined directly without approximation or assumption.

[Insert figure 6 about here]

Indentation tests were performed at a displacement rate of $0.1 \mu \mathrm{m} \mathrm{s}^{-1}, 1 \mu \mathrm{m} \mathrm{s}^{-1}$ and $5 \mu \mathrm{m} \mathrm{s}^{-1}$ up to a maximum load of approximately $1 \mathrm{~N}$, followed by unloading with the same displacement rate. Since the main interest was in the loading, no holding time at the maximum of the loading was applied.

For the load relaxation tests, the indenter penetrated into the sample at a speed of $1 \mu \mathrm{m} / \mathrm{s}$ until a maximum force of approximately $1.1 \mathrm{~N}$ was reached. The depth of penetration was then fixed at the value at the peak load during the load relaxation.

\section{Results and discussions}

\subsection{Experimental determination of the contact area}

Using the instrumented indentation microscope, the contact area was continuously recorded during indentation tests. Figure 7a shows the development of the contact area during the loading. The images were captured in-situ during indentation tests on PC. Figure $7 \mathrm{~b}$ shows the state of the deformation corresponding to the images. As expected the contact area increases gradually with an increasing indentation depth, see figure 7 a.

The diameter of the contact radius is determined from the pictures, using an image processing program. From the contact radius $a$ obtained, the contact area $A_{p}$ is subsequently calculated as $A_{p}=\pi a^{2}$, and then plotted against the penetration displacement $h$ in figure 7c. For the lower indentation depths (up to 1.8 $\mu \mathrm{m}$ ), the experimental contact area as function of the displacement shows a linear dependence, indicating an Hertzian contact (figure 7c). At deeper indentation depth, this trend gradually deviates from the linearity, indicating the occurrence of plastic deformation.

[Insert figure 7 about here]

\subsection{Numerical simulation vs. experiments}

To complete the constitutive model for our materials, the state parameter $S_{\mathrm{a}}$ must be determined in addition to the already known parameters listed in table 2 . This was done by carrying out macroscopic tensile tests on the as received and on the annealed materials. Experiments were performed at an engineering strain rate of $10^{-3} \mathrm{~s}^{-1}$ 
on samples with geometry according to ISO 527. Numerical simulations of the tensile tests were performed using the 3D tensile bar geometry and $S_{a}$ values were subsequently increased until the simulated yield stress agreed very well with the experimental one. For the as received PC a yield stress of $63 \mathrm{MPa}$ leads to a value of 31.7 for $S_{a}$. For the annealed material, a yield stress of $71 \mathrm{MPa}$ corresponds to a value of 39 for $S_{a}$. The experimental indentation tests, performed with the optical indentation microscope, were then simulated using these values for the state parameter. A direct comparison between the numerical simulation and experiments, shown in figure 8 , proves that the model can perfectly predict the experimental loaddisplacement curve (figure 8a) as well as the evolution of the experimental contact area during the indentation tests (figure 8b). At small indentation depth, the contact follows a Hertzian description, hence it is purely elastic. In contrast, at deeper indentation depth, the curves gradually deviate from the Hertzian contact due to onset of plastic deformation. In addition, it is observed that annealing leads to a shift of the load-displacement curve towards higher force at the same indentation depth and a shift towards a deeper indentation depth for the same contact area (figure 8). In general, these observations are in agreement with an extension of the elastic range due to an increase of the yield stress. Annealing leads to an increase of the yield stress and it therefore extends the elastic range.

\section{[Insert figure 8 about here]}

Since mechanical properties of glassy polymers are time-dependent, the effect of the indentation speed on the evolution of the contact area was also investigated. Simulation of indentation tests on the as-received polycarbonate at different speeds $\left(0.1 \mu \mathrm{m} \mathrm{s}^{-1}\right.$ and $\left.5 \mu \mathrm{m} \mathrm{s}^{-1}\right)$ were therefore performed and compared with the experiments. Figure 9 presents this comparison and shows that the model perfectly predicts the experimental load-displacement curve, see figure 9a, as well as the evolution of the contact area during indentation at different speed (figure 9b). Again the curves follow the Hertzian contact at low displacement, and gradually deviate at larger displacement after the onset of plastic deformation. Moreover, an increase of the speed leads to an increase of the yield stress and therefore prolonged the elastic range during the indentation.

[Insert figure 9 about here]

A difference of a factor 50 in speed leads to a difference of $5 \mathrm{MPa}$ in the yield stress between the lowest and the highest speed. Besides the difference in the yield stress between the "as received" and annealed $\mathrm{PC}$ is $8 \mathrm{MPa}$. Although the influence on the yield strength of the annealing step is more pronounced than that of the indentation speed (in the range between $0.1 \mu \mathrm{m} \mathrm{s}^{-1}$ and $5 \mu \mathrm{m} \mathrm{s}^{-1}$ ), it can be seen from a comparison between figures 8-9, that the thermal-history effect induces a slightly smaller effect on the evolution of the contact area than the speed. This agrees well with the fact that by increasing the speed the complete intrinsic response of the PC is shifted to higher stress whereas the thermal-history only induces differences in the intrinsic response close to the yield stress (see figure 2).

\subsection{Large deformation investigation: pile-up}

To further investigate the effect of the thermal history on the evolution of the contact area we performed numerical simulations of indentations to large indentation depth for two extreme values of $S_{a}$, namely 0 , which is the completely rejuvenated state, and 30, further referred as slightly annealed, for a speed of $0.1 \mu \mathrm{m}$ $\mathrm{s}^{-1}$. The difference in intrinsic behaviour between these two materials is illustrated in figure 2b. Figure 10a presents the calculated contact-area evolution during indentation for both cases. At indentation depths smaller 
than $20 \mu \mathrm{m}$ (i.e., $\mathrm{h} / \mathrm{R}<0.2$ ), the curves exhibit a similar trend as that observed in figure $8 \mathrm{~b}$, i.e. a shift of the contact area evolution towards larger depths with increasing value of $S_{a}$. Remarkably, however, this trend is inverted for larger indentation depths $(>20 \mu \mathrm{m})$, i.e. a shift of the contact area evolution towards smaller depths with increasing value of $S_{a}$.

The occurrence of this trend inversion is clearly coupled to the presence of strain softening in the case of $S_{a}=30$, and not to a simple increase of the yield stress. This is supported by the results in figure $10 \mathrm{~b}$ of simulations of indentation of the rejuvenated material $\left(S_{a}=0\right)$ at two different speeds $\left(0.1 \mu \mathrm{m} \mathrm{s}^{-1}\right.$ and $10 \mu \mathrm{m} \mathrm{s}^{-}$ $\left.{ }^{1}\right)$. The increase of indentation speed will lead to an increase of the yield stress, but for both speeds strain softening is absent $\left(S_{a}=0\right)$.

Please note, that these results were obtained assuming frictionless contact. A recent investigation into metals indicates that friction could significantly affect the indentation results when extensive pile-up occurs [30]. For polymers, this has not been verified yet.

[Insert figure 10 about here]

Since the evolution of the contact area is related to the contact geometry, i.e. pile-up or sink-in, we plot now the surface profile at the vicinity of the tip obtained for $S_{a}=0$ and $S_{a}=30$ at different indentation depths, see figure 11. This figure shows that, at low depth, the surface exhibits the same sink-in profile for both the annealed and rejuvenated material. At large indentation depth, numerical simulation shows that the annealed material piles-up where as the rejuvenated material sinks-in near the tip.

[Insert figure 11 about here]

Therefore the trend inversion in the contact area evolution observed in figure 10a, results from the difference in the contact geometry, which is determined by the post-yield behaviour. In general, strain softening will | promote the occurrence of strain localization phenomena [31]. The occurrence of pile-up for the annealed material appears to be a related phenomenon. This becomes especially clear if we look at figure 12 . This figure represents the surface profile at the maximum depth. It can be seen that for both materials, the surface will pile-up but the effect is much more pronounced for $S_{a}=30$. Moreover, even if the surface piles-up for the rejuvenated material, it does not affect the contact area evolution. Reason for that is that the first contact point between the surface and the tip ( $\mathrm{D}$ in figure 12) is lower than the free surface. Therefore, this indicates a sinkin contact which is consistent with observations from figure 11. For the slightly annealed material an opposite situation is observed. The differences between the two materials are caused by the strain softening behaviour of the annealed material that localizes the deformation close to the contact region, whereas the absence of strain softening for the fully rejuvenated sample distributes the deformation over a larger volume of the material.

[Insert figure 12 about here]

\subsection{Relaxation}

Load relaxation indentation was performed and simulated for an as-received polycarbonate sample for which no further heat treatment was applied, using the experimental conditions described in the paragraph 3.2. It should be noticed that our model takes into account only plastic effects, and no viscoelastic relaxation is incorporated. The intention here is to have an estimation of the contribution of the plasticity to stress relaxation phenomena during indentation. Figure 13 represents the simulation of the load relaxation test, using 
only a plastic model, compared with the experiments. It is shown that the simulated plastic contribution represents the quite substantial value of around $40 \%$ of the observed force relaxation.

[Insert figure 13 about here]

\section{Conclusion}

The evolution of the contact area between a spherical indenter and a polycarbonate surface was measured insitu using a specially designed instrumented indentation microscope. Indentations on PC samples with two different thermal histories were performed at two different speeds and, subsequently compared with numerical simulations carried out using an advanced elasto-plastic constitutive model for polycarbonate. The parameters used in the constitutive model were determined using tensile tests. For all cases, the numerical model perfectly predicted the development of the contact area during indentation. The results show that by annealing or by increasing the speed, the purely elastic range is extended during indentation due to the increase of the yield stress, which leads to a decrease in contact area at equal indentation depths.

Using numerical simulation, it was also shown that the post yield behaviour of the material has a dominant influence on the contact geometry, which may exhibit pile-up or sink-in. Particularly, pile-up results from localization effects and it is therefore promoted by strain softening.

Finally, in load-relaxation indentation of polycarbonate, using our model, we found that approximately half of the total relaxation is constituted by plasticity, on top of possible viscous effects.

\section{Acknowledgements}

The authors wish to acknowledge the financial support of the Netherlands Organization for Scientific Research (NWO), division chemical sciences under grant No. EPC 5745, and The Japan Society for The Promotion of Science [The Grant-in Aid for Scientific Research (B), Grant No. 18360315].

\section{References}

[1] H. Hertz, J. Reine Angew. Math. 92156 (1881)

[2] W.C. Oliver and G.M. Pharr, J Mater. Res. 71564 (1992)

[3] J.S. Field and M.V. Swain, J Mater. Res. 8297 (1993)

[4] A. Bolshakov and G.M. Pharr, J. Mat. Res. 131049 (1998)

[5] B. Taljat and G.M. Pharr, Int. J. of Solids and Structures. 413891 (2004)

[6] J. Alkorta, J.M. Martinez-Esnaola and J.G. Sevillano, J. Mater. Res. 20432 (2005)

[7] O. Casals and J. Alcala, Acta Materialia 533545 (2005)

Formatted: Portuguese (Brazil)

[8] L. Wang and S.I. Rokhlin, J. Mater. Res. 21995 (2006)

Formatted: English (U.S.)

| [9] _ M.C. Boyce, D.M. Parks, and A.S. Argon, Mech. of Mat. 715 (1988)

Deleted: 6 
| [10] E.M. Arruda and M.C. Boyce, Int. J. Plast. 9697 (1993)

| [11] O.A. Hasan and M.C. Boyce, Polym. Eng. Sci. 35331 (1995)

| [12] C.P. Buckley and D.C. Jones, Polymer. 363301 (1995)

| [13] P.J. Dooling, C.P. Buckley, and S. Hinduja, Polym. Eng. Sci. 38892 (1998)

| [14] C. Gerlach, C.P. Buckley, and D.P. Jones, Trans. Inst. Chem. Eng. Part A. 7638 (1998)

| [15] T.A. Tervoort, E.T.J. Klompen, and L.E. Govaert, J. Rheol. 40779 (1996)

| [16] L.E. Govaert, P.H.M. Timmermans, and W.A.M. Brekelmans, J. Eng. Mat. and Tech. 122 177 (2000)

| [17] E.T.J. Klompen, T.A.P. Engels, L.E. Govaert, and H.E.H. Meijer, Macromol. 386997 (2005)

| [18] T. Miyajima and M. Sakai, Phil. Mag. 865729 (2006)

| [19] M. Sakai, N. Hakiri, and T. Miyajima, J. Mat. Res. 212298 (2006)

| [20] T.A. Tervoort, R.J.M. Smit, W.A.M. Brekelmans, and L.E. Govaert, Mech. Time-Dep. Mater. 1269 (1998)

| [21] E.T.J. Klompen, T.A.P. Engels, L.C.A. van Breemen, P.J.G. Schreurs, L.E. Govaert, and H.E.H. Meijer, Macromol. 387009 (2005)

| [22] R.N. Howard and G. Thackray, Proc. Royal Soc. London A. 302 453-472 (1967)

| [23] T.A. Tervoort and L.E. Govaert, J. Rheol. 441263 (2000)

| [24] F.P.T. Baaijens, Rheol. Acta. 30284 (1991)

[25] Defined as $\bar{\tau}=\sqrt{\frac{1}{2} \operatorname{tr}\left(\boldsymbol{\sigma}_{\mathbf{s}}^{\mathrm{d}} \boldsymbol{\sigma}_{\mathbf{s}}^{\mathrm{d}}\right)}$

[26] Defined as $p=-\frac{1}{3} \operatorname{tr}\left(\boldsymbol{\sigma}_{\mathbf{s}}\right)$

| [27] I.M. Ward, J. Mater. Res. 61397 (1971)

| [28] R.A. Duckett, B.C. Goswami, L.S.A. Smith, I.M. Ward, and A.M. Zihlif, Brit. Polym. J. 1011 (1978)

[29] Defined as $\dot{\bar{\gamma}}_{p}=\sqrt{\operatorname{tr}\left(\mathbf{D}_{\mathbf{p}} \mathbf{D}_{\mathbf{p}}\right)}$

[30] M. Mata and J. Alcala, J. Mech. Phys. Solids 52145 (2004)
Deleted: 7

Deleted: 8

Deleted: 9

Deleted: 0

Deleted: 1

Deleted: 12

Deleted: 3

Deleted: 4

Deleted: 5

Deleted: 6

Deleted: 17

Deleted: 18

Deleted: 19

Deleted: av

Deleted: 20

Formatted: Dutch (Netherlands)

Deleted: 1

Deleted: 2

Deleted: 3

\section{Deleted: 4}

Deleted: 5

Deleted: 6

Formatted: English (U.S.)

Formatted: English (U.S.)

Formatted: Portuguese (Brazil) 
[Figure captions]

Figure 1. Contact profile developed during indentation where $h$ is the indentation depth, $h_{c}$ is the contact depth, $a$ is the contact radius.

Figure 2. Intrinsic behavior of amorphous polymer: (a) under the influence of different true strain rates and; (b) at different thermal histories.

Figure 3. (a) Influence of the thermal history and strain softening on the strain rate dependence of the yield stress; (b) intrinsic stress-strain curve indicating the influence of the physical aging.

Figure 4. Compression tests: (a) experiments (open symbols) compared with the numerical simulation (solid lines) using material parameters presented in table 1 for three different true strain rates: $10^{-2} \mathrm{~s}^{-1}(\square), 10^{-3} \mathrm{~s}^{-1}(0), 10^{-4} \mathrm{~s}^{-1}(\nabla)$; (b) simulated compression tests (solid lines) for two different values of the elastic modulus $(\mathrm{E}=900 \mathrm{MPa}$ and $\mathrm{E}=2.2 \mathrm{MPa}$ ) compared with the experiments (O).

Figure 5. Finite element mesh used to simulate the indentation tests.

Figure 6. Instrumented indentation microscope set up.

figure 7. Contact area measurements: (a) pictures of the contact area obtained in situ during indentation tests; (b) load displacement curve indicating at which state of the deformation the pictures were recorded; (c) experimental contact area ( $\square$ ), determined from the pictures, as function of the indentation depth. The solid line corresponds to a Hertzian contact.

Figure 8. Indentation experiments (open symbols) compared with the numerical simulation (solid line) for two different thermal histories: annealed (ㅁ) and as received (O): (a) for the load-displacement and; (b) for the contact area normalized with the square of the tip radius as function of the displacement normalized with the radius $(100 \mu \mathrm{m})$.

Figure 9. Indentation experiments (open symbols) compared with the numerical simulation (solid line) for two different speeds: $\dot{h}=0.1 \mu \mathrm{m} \mathrm{s}^{-1}$ (口) and $5 \mu \mathrm{m} \mathrm{s}^{-1}$ (O): (a) for the load-displacement and; (b) for the contact area normalized with the square of the tip radius as a function of the displacement normalized with the radius.

Figure 10. Normalized contact area as function of the normalized indentation depth: (a) for two different thermal-histories modelled by two different $S_{a}$ values: 0 (rejuvenated) for the solid line and 30 (annealed) for the dashed line; (b) for two different speeds $\left(S_{a}=0\right): 10 \mu \mathrm{m} \mathrm{s}^{-1}$ for the solid line and $0.1 \mu \mathrm{m} \mathrm{s}^{-1}$ for the dashed line.

Figure 11. Contact profile for the slightly annealed material (solid line) and the rejuvenated material (dashed line) at different indentation depths $(12.5 \mu \mathrm{m}$ for profile number $1,25 \mu \mathrm{m}$ for number $2,37.5 \mu \mathrm{m}$ for number 3 and $50 \mu \mathrm{m}$ for number 4$)$.

Figure 12. Simulated contact profile as function of the radial distance from the center: for the rejuvenated PC (dashed line) and slightly annealed PC (solid line) at an indentation depth of $50 \mu \mathrm{m}$. The dotted line on the left represents the tip profile.

Figure 13. Experimental indentation load relaxation test result $(+)$ compared with the simulation (solid line) using a model which incorporates only plasticity, but no viscoelasticity. 
[Table captions]

Table 1. Material parameters used for the numerical simulation of tensile and compression tests on PC.

Table 2. Material parameters used for the numerical simulation of indentation tests on PC. 
[Table 1]

\begin{tabular}{|c|c|c|c|c|c|c|}
\hline$K[\mathrm{MPa}]$ & $G$ [MPa] & $G_{r}[\mathrm{MPa}]$ & $\eta_{o, r}[\mathrm{MPa} \mathrm{s}]$ & $\tau_{o}[\mathrm{MPa}]$ & $\mu$ & $S_{a}[-]$ \\
\hline 3750 & 321 & 26 & $2.1 .10^{11}$ & 0.7 & 0.08 & - \\
\hline
\end{tabular}


[Table 2]

\begin{tabular}{ccccccc} 
& & & & & \\
\cline { 2 - 4 }$[\mathrm{MPa}]$ & $G[\mathrm{MPa}]$ & $G_{r}[\mathrm{MPa}]$ & $\eta_{o, r}[\mathrm{MPa} \mathrm{s}]$ & $\tau_{o}[\mathrm{MPa}]$ & $\mu$ & $S_{a}[-]$ \\
\hline 3750 & 784 & 26 & $2.8 .10^{12}$ & 0.7 & 0.08 & -
\end{tabular}


[Figure 1]

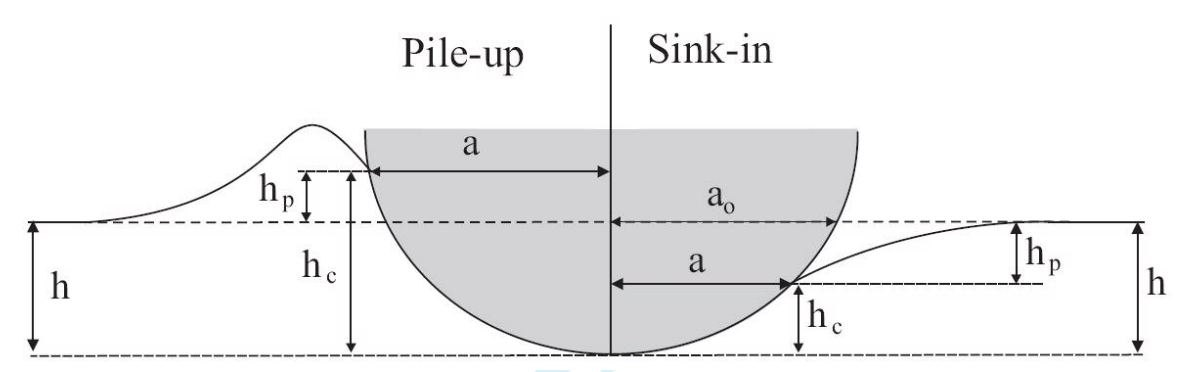


[Figure 2]

(a)

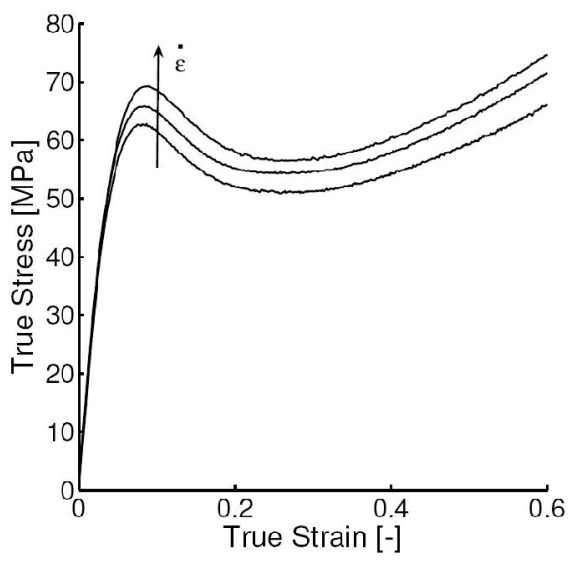

(b)

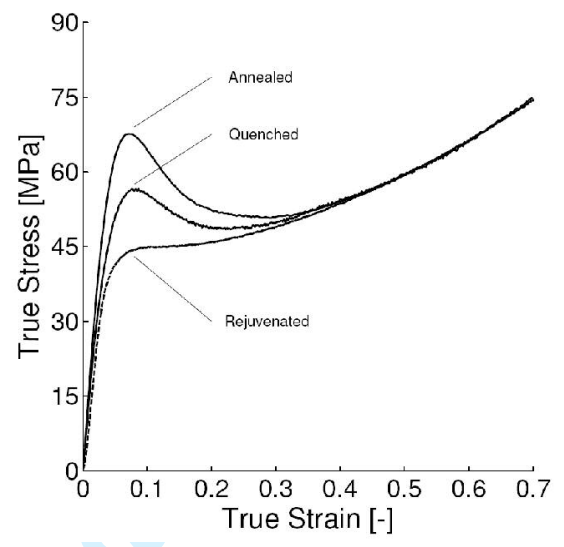


[Figure 3]

(a)

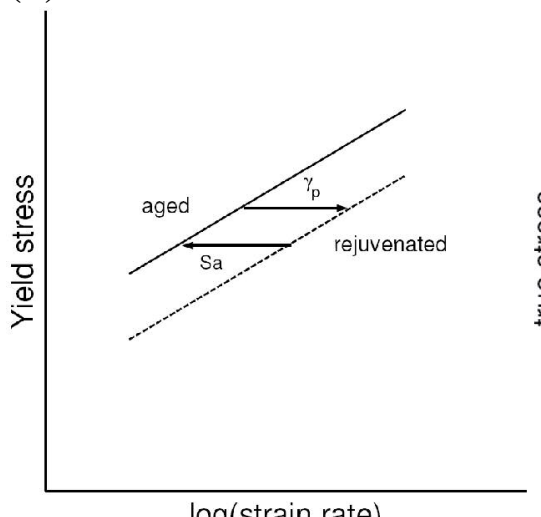

$\log$ (strain rate) (b)

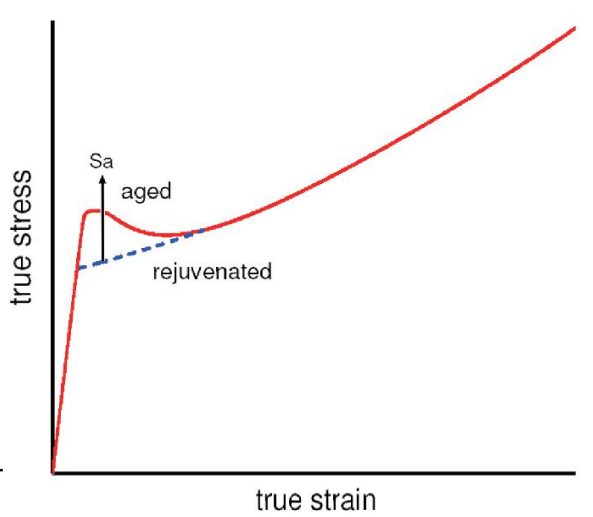


[Figure 4]

(a)

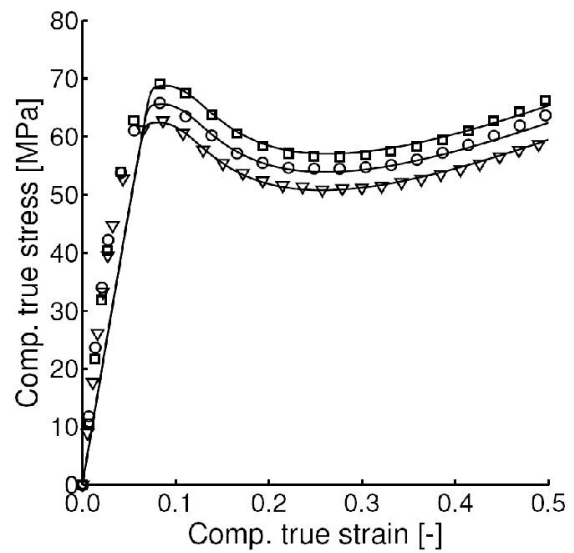

(b)

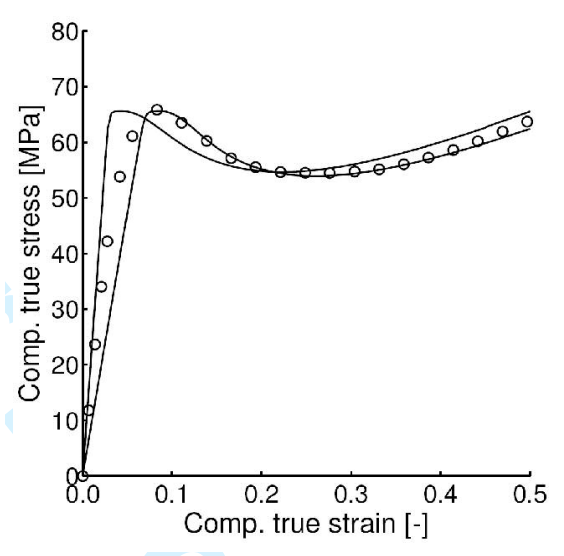


[Figure 5]

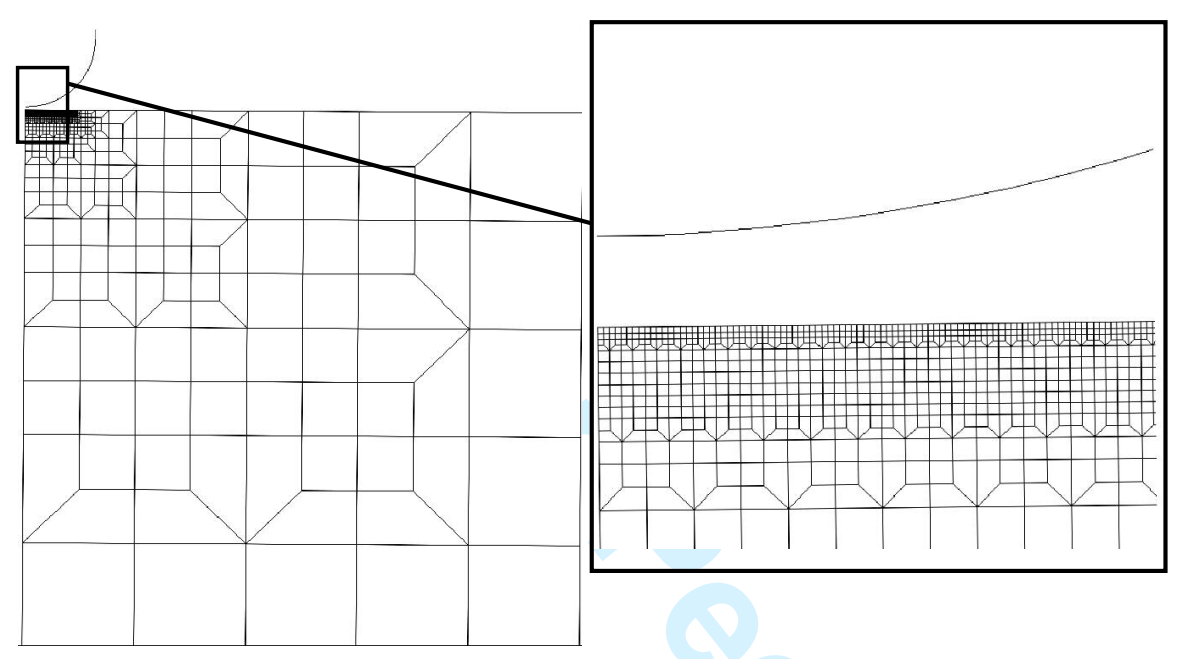


[Figure 6]

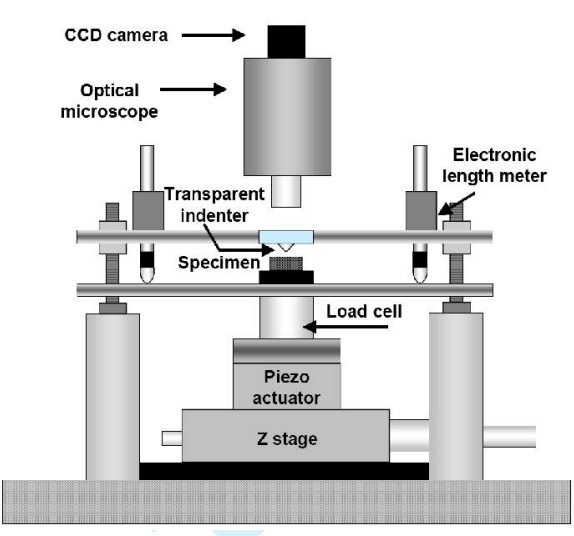

http://mc.manuscriptcentral.com/pm-pml 


\section{Page 23 of 29}

[Figure 7]

(a)
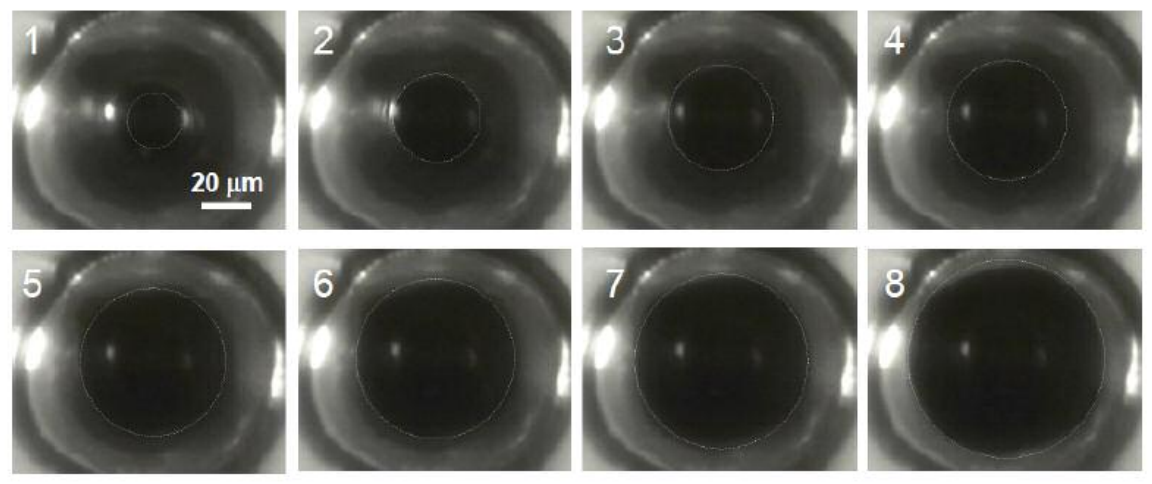

(b)

(c)
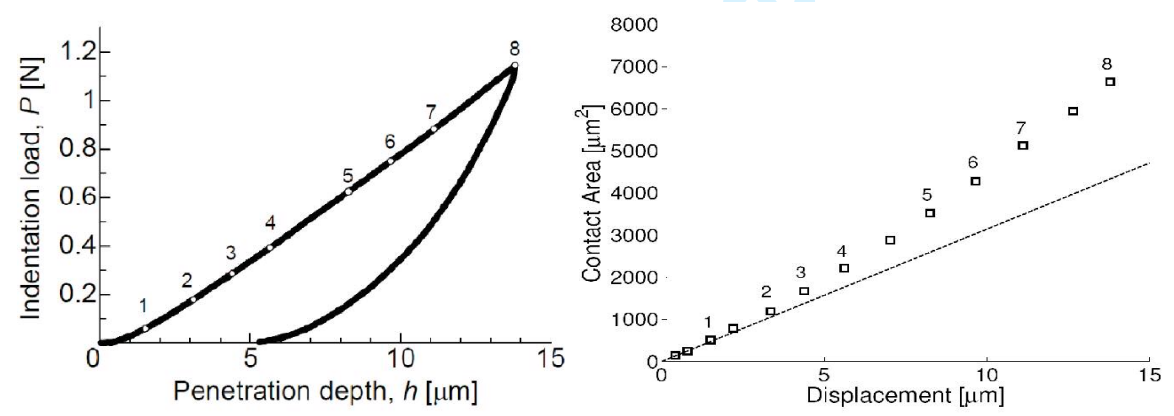
[Figure 8]

(a)

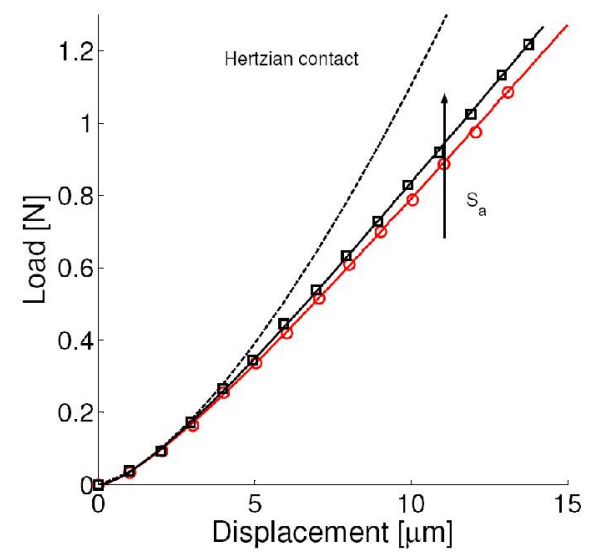

(b)

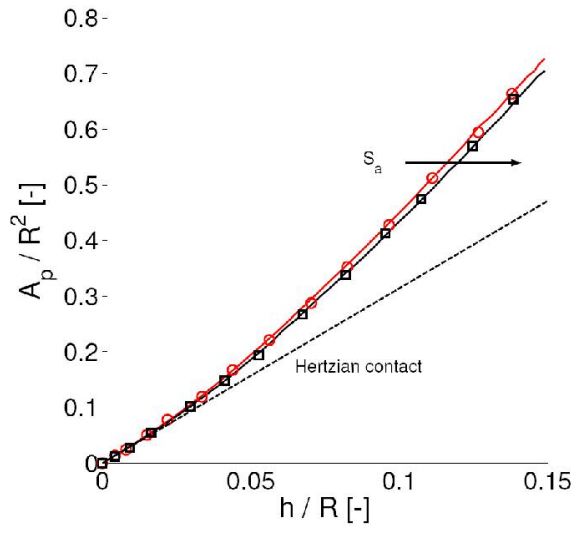


[Figure 9]

(a)

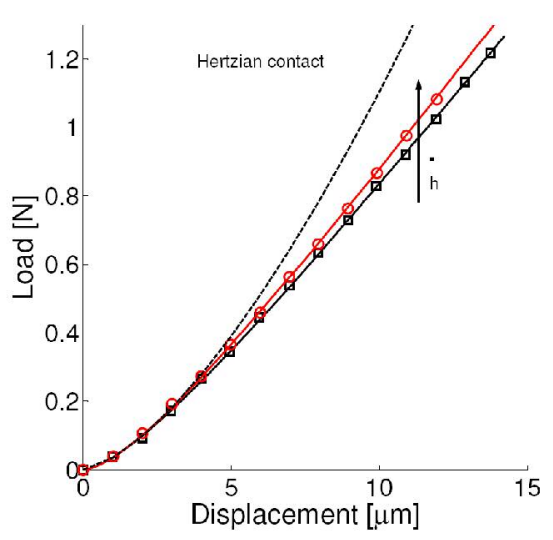

(b)

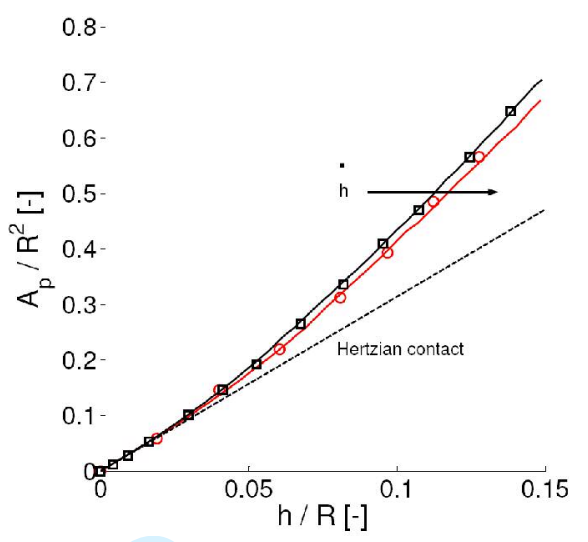


[Figure 10]

(a)

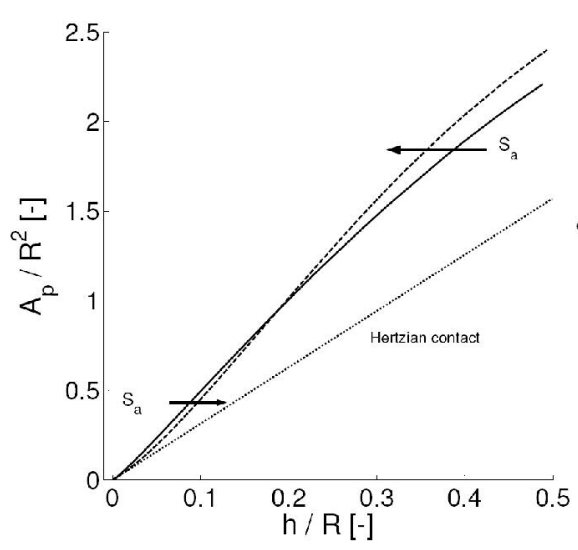

(b)

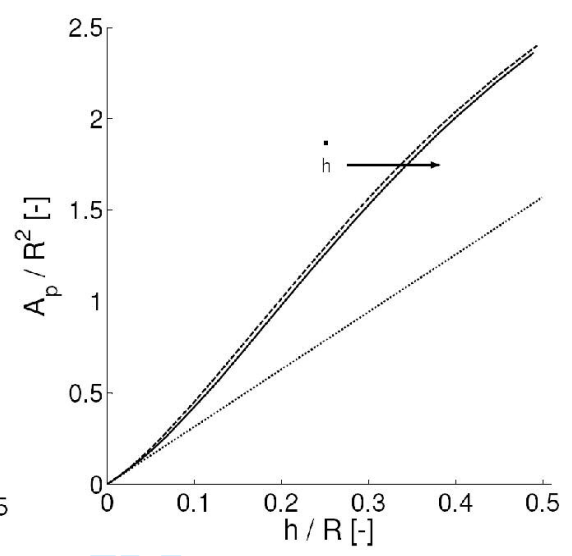


[Figure 11]

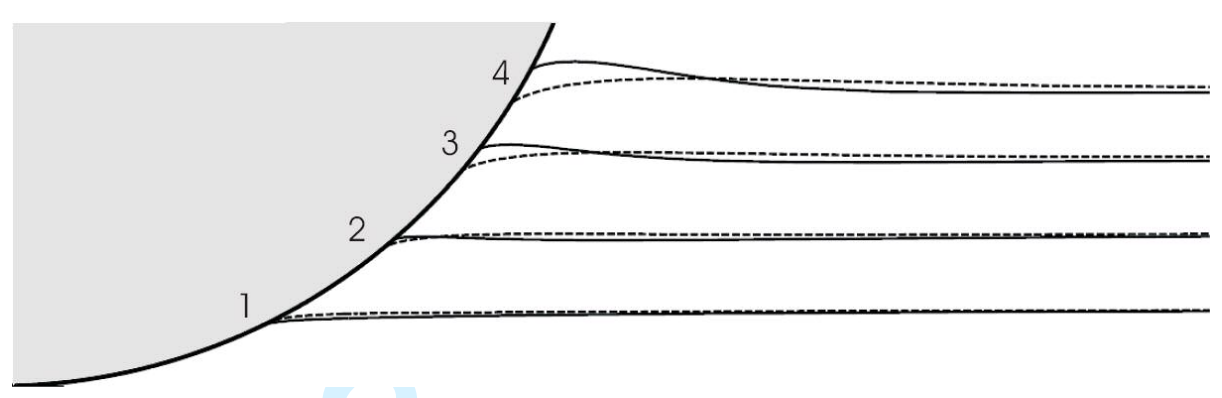


[Figure 12]

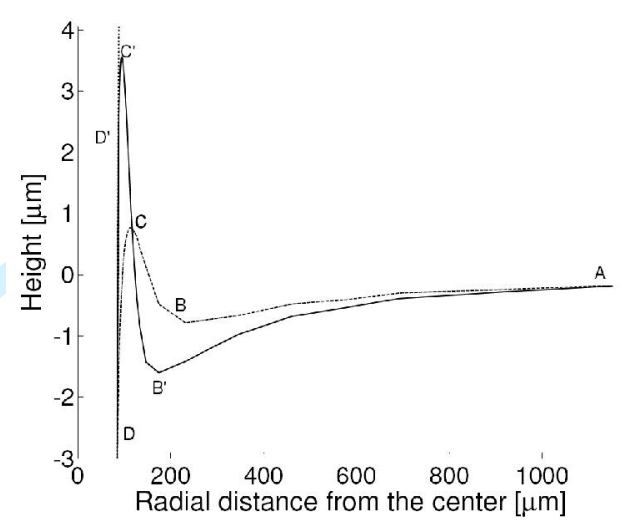


[Figure 13]

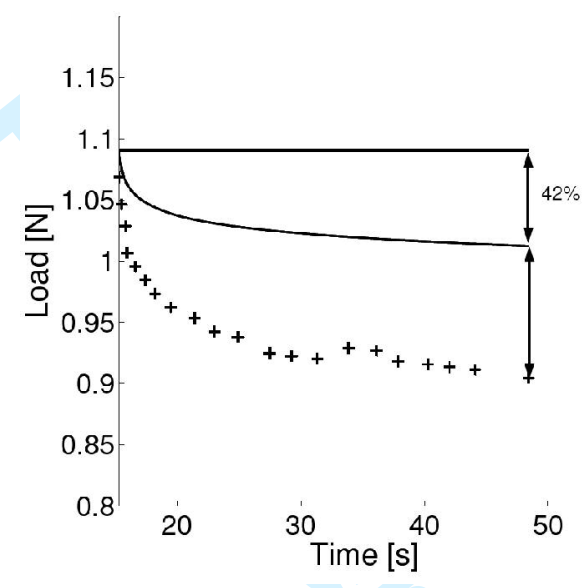

http://mc.manuscriptcentral.com/pm-pml 